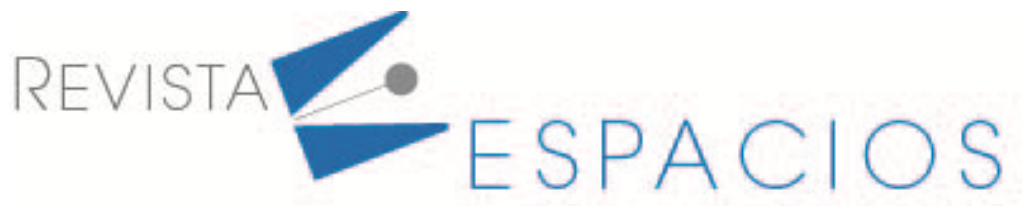

Vol. 42 (14) $2021 \cdot$ Art. 1

\title{
El deterioro de los inventarios y la disminución de la utilidad neta en las empresas industriales del Perú
}

\section{The impairment of inventories and the decrease in net income in industrial companies in Peru}

TORRES, Gustavo A. ${ }^{1}$

\begin{abstract}
Resumen
El objetivo de la investigación fue analizar el deterioro de los inventarios y la relación con los resultados económicos de las empresas, lo cual implicó el desarrollo de la investigación descriptiva. Los resultados, nos muestran que existe significativa relación entre el deterioro de inventarios y la disminución de la utilidad neta de las empresas industriales de Perú.

Palabras clave: deterioro de inventarios, utilidad neta

Abstract

The objective of the research was to analyze the impairment of inventories and the relationship with the economic results of the companies, which implied the development of descriptive research. The results show us that there is a significant relationship between the impairment of inventories and the decrease in the net profit of industrial companies in Peru.

key words: impairment of inventory, net profit
\end{abstract}

\section{Introducción}

\subsection{Aspectos generales}

El deterioro del valor de los inventarios en los almacenes de las empresas se origina por diversas causas, que forman parte del presente estudio, cuyos efectos se expresan en la disminución del valor monetario de los inventarios y las pérdidas que se reflejan en el Estado de Resultados.

En ese sentido, el objetivo de la investigación fue, analizar el deterioro de los inventarios y la relación con los resultados económicos de las empresas industriales del Perú, lo que implicó el desarrollo de la investigación descriptiva.

La Norma Internacional de Contabilidad No. 2 (NIC-2 Inventarios) señala situaciones en las cuales los costos no pueden ser recuperados, por estar dañados, obsoletos o los precios del mercado han caido. Asimismo, el costo de los inventarios puede no ser recuperable si los costos estimados para su terminación o su venta han aumentado. La práctica de rebajar el saldo, hasta que el costo sea igual al valor neto realizable, es coherente con

\footnotetext{
${ }^{1}$ Universidad Nacional del Altiplano Puno. Perú. gustavot@unap.edu.pe .ORCID:0000-0002-8438-1159. Profesor en la Escuela Profesional de Ciencias Contables. epcontables@unap.edu.pe
} 
el punto de vista según el cual los activos no deben registrarse en libros por encima de los importes que se espera obtener a través de su venta o uso. Por tanto, la hipótesis fue:

El deterioro de los inventarios incide en los resultados económicos de las empresas industriales de Perú, en 2017 $-2019$.

Los resultados nos permitieron conocer cómo incide la norma internacional de contabilidad N² -Inventarios, en la aplicación y determinación del deterioro de existencias en las empresas industriales del Perú.

Los inversionistas debieran considerar el deterioro de los inventarios como un porcentaje menor de utilidad neta recibida, para un acertado análisis de los estados financieros. El deterioro de los inventarios, generalmente está relacionado con la gestión de la empresa, la cual es responsabilidad de la alta dirección.

El área de producción puede ser el responsable del deterioro de inventarios, cuando se utilizan materiales a punto de vencer, según la vida útil proyectada o por trabajar con materiales de baja calidad. Cuando se trate de asuntos de marketing, se tendría que considerar lo que se vendió y la utilidad (ganancia) que generó contra el importe deteriorado, para determinar el grado de éxito de la campaña de marketing.

El deterioro de los inventarios también puede tener origen en la gestión logística, cuando se mantiene exceso de mercaderías en los almacenes y terminan por caducar o pasar de moda, o por una inadecuada manipulación de los inventarios.

Lo descrito es parte de la vida íntima de una empresa, muchas veces no se llega a conocer si no se analiza el origen del deterioro de los inventarios y cuánto afectó a la utilidad neta. En Perú, las mermas de producción; forman parte del costo del producto terminado, siempre que se fundamente en estudios técnicos, en ese sentido, los importes del deterioro de inventarios presentados, no incluyen las mermas de producción.

Las crisis económicas, por lo general se relacionan con el incremento del desempleo y por consiguiente la disminución de la capacidad adquisitiva y ello aumenta si convivimos en pandemia, en la cual se restringe el movimiento de las personas. Las mercancías y productos rotarán a menor velocidad. Los precios de los productos son rebajados, para lograr mantener las ventas, sin embargo, existen otros casos del deterioro del inventario como detalla, el párrafo 2.28 en The International Financial Reporting Standards Foundation (IFRS Foundation, 2020):

"El costo de los inventarios puede no ser recuperable en caso de que los mismos estén dañados, si han devenido parcial o totalmente obsoletos, o bien si sus precios de mercado han caído. Asimismo, el costo de los inventarios puede no ser recuperable si los costos estimados para su terminación o su venta han aumentado. La práctica de rebajar el saldo, hasta que el costo sea igual al valor neto realizable, es coherente con el punto de vista según el cual los activos no deben registrarse en libros por encima de los importes que se espera obtener a través de su venta o uso".

Soto-Restrepo, Quiros-Jaramillo y Mesa-Velásquez (2014.) coinciden en que: "El costo de los inventarios puede no ser recuperable al estar dañados, si han devenido parcial o totalmente obsoletos, o si sus precios de mercado han caído".

La principal causa de que los productos se vendan a menor precio y se genere el deterioro de valor de los inventarios, se debe al decrecimiento de la demanda, según Valdivieso, (2020), se aprecia que: "La disminución de la demanda origina la reducción de los precios en el mercado; en cuanto a inventarios, estos sufren deterioro de su valor, por lo que las empresas se ven forzadas a reducir los precios y rotar de inmediato sus inventarios, como medida de contención a la crisis económica". 
Si la empresa tuviera inventarios deteriorados y no efectúan el ajuste pertinente, se configura la sobrevaloración de los inventarios. Benavides (2017), informa que : "Se evidenció que los inventarios de la empresa Electro Irmavi se encontraban sobrevalorados, al mantener productos deteriorados como la grifería, baldosas, emporador y bondexcon. Los deterioros de los inventarios se originaron por defectos de fábrica, también por daños ocasionados en la manipulación y transporte".

El deterioro de valor de los inventario, también se le denomina "desmedro", como señala, Ferrer (2010), el cual describe los siguientes ejemplos: "Las medicinas que tengan sus fechas de uso vencidas, las prendas de vestir que por el tiempo hayan pasado de moda, las máquinas de escribir al quedar en desuso, los disquetes con los CD; y posteriormente, este último fue superado por la aparición del USB".

El deterioro de valor de los inventarios hay que medirlo, en efecto, Campoverde y Orellana (2012) concluyen que:

"Si los inventarios sufren un deterioro por obsolescencia, daños o cambio tecnológico; se tienen que medir al menor costo entre su importe en libros y su valor neto realizable".

El deterioro de los inventarios afecta a los resultados económicos, debido al reconocimiento como gasto y la disminución del rubro de las existencias.

Jiménez y Rivera, (2019) concluyen que las mermas y desmedros impactan en lo que respecta a la situación financiera y tributaria en las empresas del sector manufactura (actividades de impresión) en la Zona Este y Lima, 2017 , como se indica en el siguiente párrafo:

"Las empresas del sector manufactura, específicamente en el subsector de actividades de impresión, usualmente registran las mermas con los porcentajes generales de la industria y no calculan minuciosamente el valor real de las mermas, lo que ocasiona que no tengan costos exactos del bien producido y gastos relacionados, lo cual distorsiona los estados financieros".

Desde el punto de vista de la tributación peruana, los desmedros de los inventarios, requieren el cumplimiento de estrictos procedimientos, tal como detalla, Abanto (2016), que considera dos casos del deterioro de los inventarios: las obsoletas y los desmedros; específicamente señala: "De acuerdo con el numeral 2 del literal c) del artículo 21 del Reglamento de la Ley del Impuesto a la Renta, el desmedro es la pérdida de orden cualitativo e irrecuperable de las existencias, haciéndolas inutilizables para los fines a los que estaban destinados. Sin embargo, pueden ser deducibles en el ejercicio en que se produzca alguna de las siguientes situaciones:

a) Mediante la destrucción de los bienes y se comunique a la Sunat (Superintendencia Nacional de Administración Tributaria) tal destrucción en un plazo no menor de seis (6) días hábiles anteriores a la fecha en que se lleve a cabo, ante la presencia de un notario público o juez de paz, a falta de aquel; pudiendo la Sunat designar a un funcionario para presenciar dicho acto. b) Se venda o entregue como dación en pago,.c) Su donación.

Conforme con lo anterior, se advierte que desde el enfoque del balance general dispuesto en la Norma Internacional de Contabilidad 12 (NIC 12), se generará un impuesto diferido, que deberá ser reconocido por la empresa, toda vez que cumplido alguna de las situaciones antes descritas, la empresa podrá reconocer la pérdida deduciendo su renta neta imponible en dicho ejercicio".

En Perú, no se acepta el gasto tributario del deterioro de los inventarios, salvo de productos alimenticios y otros que se puedan destruir, ante un Notario Público, que certifica el cumplimiento de la normativa. El desfase de la ropa fuera de la estación de moda o la obsolescencia de otros bienes, resulta complicado sustentar el gasto tributario. 
Garizabal, Barrios, Bernal y Garizabal (2020) afirman que las empresas "vienen declarando inventarios que se encuentran en mal estado y que contablemente están provisionados en un $100 \%$. Al determinar el valor patrimonial de los inventarios, la provisión no es aceptada fiscalmente como deducción, ni como disminución del valor patrimonial a los mismos; incrementando el patrimonio líquido a declarar en cada año. (Hasta el 2016). A partir de 2017 aplica el art. 64 del Estatuto Tributario".

La pérdida de inventarios por robos o hurtos, no es parte del deterioro de los inventarios, son hechos delictivos que siguen caminos diferentes, como son los procesos judiciales o la solicitud de la cobertura a las empresas de seguros, en ese sentido, discrepo con: Bernia, Brancacho y Galarza, (2017.): en sus conclusiones sobre los conceptos incluidos en la desvalorización de los inventarios, que inciden en la rentabilidad de las empresas:

"En consecuencia, se tiene una gran cantidad de desperdicio, robos o hurtos e incidiendo contablemente y financieramente.

Incide en el estado de situación financiera, por la baja de existencias por desperdicios, robo o hurto de materiales de construcción"

En ese sentido, Fonseca y Murillo (2015), sostienen que "incide principalmente en la determinación de los resultados a un valor razonable y del inventario final". También, Jiménez y Rivera (2019), concluyen que impactan en lo que respecta a la situación financiera en las empresas del sector manufactura.

La situación planteada sirve de base para proponer un estudio sobre el deterioro de valor de los inventarios y su efecto en los resultados de las empresas industriales del Perú, en el lapso 2017-2019, Asi como conocer la incidencia de la norma internacional de contabilidad N² -Inventarios, en la aplicación y determinación del deterioro de existencias en las empresas industriales.

\subsection{Secuencia práctica de aplicación del deterioro de los inventarios}

Las empresas se pueden perjudicar por la disminución de los precios de venta de sus inventarios, cuando tienen en almacenes el stock, y en esas circunstancias deben aplicar la prueba o test del valor neto de realización (VNR), lo cual requiere disponer de tres estimaciones, según señala Cuzcano (2020), "el precio estimado de venta, el costo estimado para terminar la producción y el costo estimado para concretar la venta".

En el gráfico 1 se muestran los procedimientos para determinar el deterioro del valor de inventarios: 


\section{Gráfico 1}

Procedimientos para determinar el deterioro del valor de inventarios. (VNR)

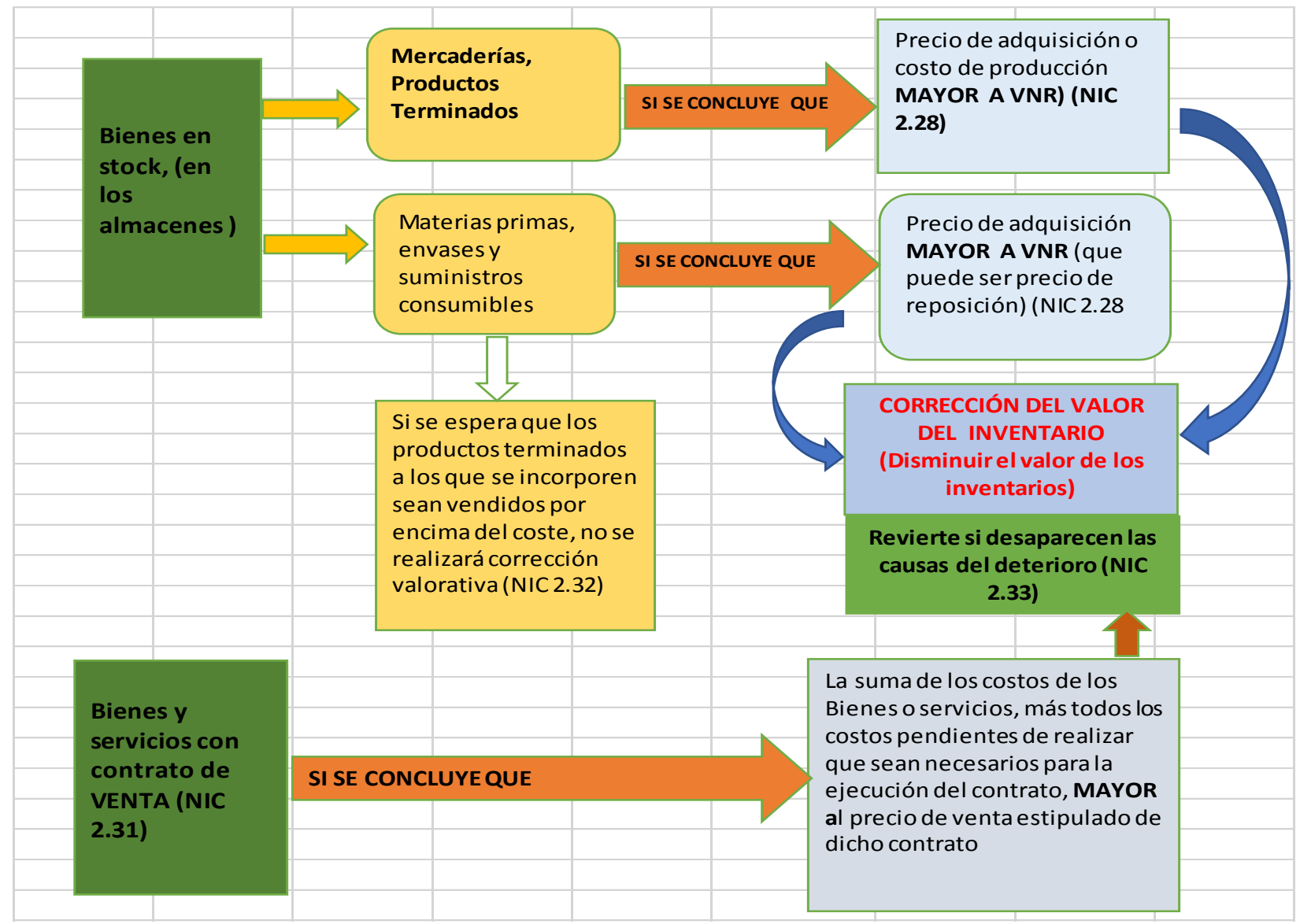

Fuente: Del Campo (2019). Contabilidad Financiera. (Con modificaciones realizadas por el autor).

La Norma Internacional de Contabilidad $N^{\circ} 2-$ Inventarios (The International Financial Reporting Standards Foundation-IFRS Foundation, 2020a), detalla una serie de definiciones sobre los costos y de la comparación con el valor neto realizable (VNR):

"El costo de los inventarios comprenderá todos los costos derivados de su adquisición y transformación, así como otros costos en los que se haya incurrido para darles su condición y ubicación actuales". (NIC 2- párrafo 10).

“Los costos de adquisición de los inventarios comprenderá el precio de compra, los aranceles de importación y otros impuestos (que no sean recuperables posteriormente de las autoridades fiscales) y transporte, manejo y otros costos directamente atribuibles a la adquisición de mercaderías, materiales y servicios. Los descuentos comerciales, las rebajas y otras partidas similares se deducirán para determinar el costo de adquisición". (NIC 2párrafo 11).

En la Norma Internacional de Contabilidad N² - Inventarios, (IFRS Foundation, 2020a) se detalla sobre el deterioro de los inventarios:

"El costo de los inventarios puede no ser recuperable en caso de que los mismos estén dañados, si han devenido parcial o totalmente obsoletos, o bien si sus precios de mercado han caído. Asimismo, el costo de los inventarios puede no ser recuperable si los costos estimados para su terminación o su venta ha aumentado. La práctica de rebajar el saldo, hasta que el costo sea igual al valor neto realizable, es coherente con el punto de 
vista según el cual los activos no deben registrarse en libros por encima de los importes que se espera obtener a través de su venta o uso". (NIC 2- párrafo 28).

"Generalmente, la rebaja hasta alcanzar el valor neto realizable se calcula para cada partida de los inventarios. En algunas circunstancias, sin embargo, puede resultar apropiado agrupar partidas similares o relacionadas. Este puede ser el caso de las partidas de inventarios relacionados con la misma línea de productos, que tienen propósitos o usos finales similares, se producen y venden en la misma área geográfica y no pueden ser, por razones prácticas, evaluados separadamente de otras partidas de la misma línea. No es apropiado realizar las rebajas a partir de partidas que reflejen clasificaciones completas de los inventarios, por ejemplo, sobre la totalidad de los productos terminados, o sobre todos los inventarios en un segmento de operación determinado". (NIC 2- párrafo 29).

La regla de costo o valor neto realizable, "el menor", según Guajardo y Andrade de Guajardo, (2019) se aplica en dos metodologías: por partidas o por categorías. (págs. 381 y382).

A continuación, presentamos las dos formas para determinar el ajuste del deterioro de valor de los inventarios. Para ambos casos, se debe calcular el valor neto realizable (VNR) el cual se presenta en la tabla 1. En la tabla 2 (método de partida por partida o por cada mercadería) y en la tabla 3 (método por categorías o por grupos similares de mercaderías), se incluyen las columnas de ajustes, de manera que se puede reconocer el asiento contable, según el método elegido.

Tabla 1

Cálculo del valor neto realizable

\begin{tabular}{|c|c|c|c|c|c|}
\hline DETALLE & \begin{tabular}{|l|} 
Lista de \\
precios de \\
venta \\
(empresa).(\$) \\
(1)
\end{tabular} & $\begin{array}{l}\text { Descuento a } \\
\text { lista de } \\
\text { precios de } \\
\text { venta (\$). (2) }\end{array}$ & $\begin{array}{l}\text { Gastos para } \\
\text { terminar de } \\
\text { producir los } \\
\text { productos } \\
\text { (\$). (3) }\end{array}$ & $\begin{array}{l}\text { Gastos de } \\
\text { marketing y } \\
\text { de ventas (\$). } \\
\text { (4) }\end{array}$ & $\begin{array}{l}\text { Valor Neto } \\
\text { Realizable } \\
\text { (VNR) (\$). (5) } \\
=(1)-(2)-(3)- \\
(4)\end{array}$ \\
\hline \multicolumn{6}{|c|}{$\begin{array}{l}\text { Grupo I (Línea de } \\
\text { productos similares) }\end{array}$} \\
\hline$x$ & 33.0 & -3.0 & -6.0 & -4.0 & 20.0 \\
\hline$Y$ & 44.0 & -4.0 & -8.0 & -4.5 & 27.5 \\
\hline Z & 40.0 & & -6.0 & -4.0 & 30.0 \\
\hline \multicolumn{6}{|c|}{$\begin{array}{l}\text { Grupo II (Línea de } \\
\text { productos similares) }\end{array}$} \\
\hline$A$ & 25.0 & & -7.0 & -4.0 & 14.0 \\
\hline B & 23.0 & & -6.0 & -5.0 & 12.0 \\
\hline
\end{tabular}

Fuente: Los autores

Los grupos están conformados por líneas de productos similares, por ejemplo, la línea de harinas, y la de precocidos, (alimentos para consumo humano), o la línea de balanceados (alimentos para consumo de los animales).

Utilizando el listado de precios de venta de los productos o últimas facturas de compras, se puede iniciar el proceso del cálculo del VNR y determinación del deterioro de los inventarios, si fuera el caso. Los porcentajes de gastos de ventas o descuentos históricos, pueden ser útiles para calcular el valor neto de realización. 


\section{a. Método de partida por partida}

Tabla 2

Método de partida por partida (por cada mercadería).

$\mathrm{VNR}=$; NIC $(2.29)=$

\begin{tabular}{|c|c|c|c|c|c|c|c|}
\hline Detalle & \begin{tabular}{|l|} 
UNIDADES \\
(Inventario). \\
(31 \\
diciembre.XX \\
). (1) \\
\end{tabular} & $\begin{array}{l}\text { Costo de } \\
\text { adquisición o } \\
\text { producción } \\
(\$) .(2)\end{array}$ & \begin{tabular}{|l} 
Valor Neto \\
Realizable \\
(VNR) (\$). (3)
\end{tabular} & \begin{tabular}{|l|} 
El menor de \\
costo VS. \\
VNR (\$). (4) = \\
$(1) X(2)$ ó (1) \\
$x(3)$ \\
\end{tabular} & $\begin{array}{l}\text { Total al valor } \\
\text { de costo (\$). } \\
(5)=(1) \times(2)\end{array}$ & \begin{tabular}{|l} 
Ajuste por \\
deterioro (\$). \\
$(6)=(5)-(4)$
\end{tabular} & $\begin{array}{l}\text { Ajustar } \\
\text { cuando: }\end{array}$ \\
\hline \multicolumn{8}{|c|}{\begin{tabular}{|l|} 
Grupo I (Línea de \\
productos similares)
\end{tabular}} \\
\hline$x$ & 400 & 25 & 20.0 & 8,000 & 10,000 & 2,000 & Costo >VNR \\
\hline $\mathrm{Y}$ & 300 & 27 & 27.5 & 8,100 & 8,100 & 0 & \\
\hline z & 200 & 24 & 30.0 & 4,800 & 4,800 & 0 & \\
\hline \multicolumn{8}{|c|}{$\begin{array}{l}\text { Grupo II (Línea de } \\
\text { productos similares) }\end{array}$} \\
\hline A & 500 & 15 & 14.0 & 7,000 & 7,500 & 500 & Costo $>$ VNR \\
\hline $\mathrm{B}$ & 600 & 13 & 12.0 & 7,200 & 7,800 & 600 & Costo $>$ VNR \\
\hline TOTAL & & & & 35,100 & 38,200 & 3,100 & \\
\hline
\end{tabular}

Nota: El ajuste es por cada mercadería

Fuente: Los autores

Se sumó una columna para el cálculo del ajuste: $(6)=,(X=2,000),(A=500) ;(B=600)$; Total $(3,100)$. (El importe total del ajuste, refleja el deterioro de los inventarios, que se tramitará mediante el asiento contable correpondiente).

La valoración depende, por lo general, de la respuesta del mercado. Cálculo en el cual se anota el valor menor entre el costo y el VNR. En el ejemplo, el costo o VNR unitario aplicado, figura en color rojo.

\section{b. Método de categorías}

En este caso, elegimos el resultado del subtotal por categoría (grupo), del ajuste del deterioro de los inventarios (Columna 8)

Tabla 3

Método de categorías (por grupos de mercaderías).

$\mathrm{VNR}=$; NIC (2.29)=

\begin{tabular}{|c|c|c|c|c|c|c|c|c|c|}
\hline Detalle & $\begin{array}{l}\text { UNIDADES } \\
\text { (Inventario). } \\
\text { (31 } \\
\text { diciembre.XX } \\
\text { ). (1) }\end{array}$ & $\begin{array}{l}\text { Costo de } \\
\text { adquisición o } \\
\text { producción } \\
\text { (\$). (2) }\end{array}$ & \begin{tabular}{|l|} 
Valor Neto \\
Realizable \\
(VNR) (\$). (3)
\end{tabular} & \begin{tabular}{|l|} 
Costo \\
histórico \\
total (\$). (4) $=$ \\
$(1) \times(2)$
\end{tabular} & $\begin{array}{l}\text { VNR Total (\$). } \\
(5)=(1) \times(3)\end{array}$ & $\begin{array}{l}\text { El menor de } \\
\text { costo VS. } \\
\text { VNR (\$). (6) }\end{array}$ & $\begin{array}{l}\text { Total al valor } \\
\text { de costo }(\$) . \\
(7)=(4)\end{array}$ & \begin{tabular}{|l|} 
Ajuste por \\
deterioro (\$). \\
$(8)=(7)$ - (5)
\end{tabular} & $\begin{array}{l}\text { Ajustar } \\
\text { cuando: }\end{array}$ \\
\hline \multicolumn{10}{|l|}{\begin{tabular}{|l|} 
Grupo I (Línea de \\
productos similares)
\end{tabular}} \\
\hline $\mathrm{x}$ & 400 & 25 & 20.0 & 10,000 & 8,000 & & 10,000 & 2,000 & \\
\hline Y & 300 & 27 & 27.5 & 8,100 & 8,250 & & 8,100 & -150 & \\
\hline $\mathrm{z}$ & 200 & 29 & 30.0 & 5,800 & 6,000 & & 5,800 & -200 & \\
\hline SUBTOTAL & & & & 23,900 & 22,250 & 22,250 & & 1,650 & Costo $>$ VNR \\
\hline \multicolumn{10}{|l|}{$\begin{array}{l}\text { Grupo II (Línea de } \\
\text { productos similares) }\end{array}$} \\
\hline A & 500 & 15 & 14.0 & 7,500 & 7,000 & & 7,500 & 500 & \\
\hline B & 600 & 13 & 12.0 & 7,800 & 7,200 & & 7,800 & 600 & \\
\hline SUBTOTAL & & & & 15,300 & 14,200 & 14,200 & & 1,100 & Costo $>$ VNR \\
\hline TOTAL MERCADERÍAS & & & & 39,200 & 36,450 & 36,450 & 39,200 & & \\
\hline \multirow[t]{2}{*}{ TOTAL AJUSTE } & & & & & & 2,750 & & 2,750 & \\
\hline & & & & & & 39,200 & & & \\
\hline
\end{tabular}

Nota: El ajuste es por cada agrupación de mercaderías (Grupos I y II)

Fuente: Los autores 
Se agregó una columna para el ajuste por Grupos: (8), (I=1650), (Grupo II=1 100); Total $(2,750)$. (El importe total del ajuste, refleja el deterioro de los inventarios, que se tramitará mediante el asiento contable correpondiente).

Ataupillco, Agurto y De la Cruz (2020) afirman que los inventarios que se encuentren totalmente deteriorados, no tienen valor, su VNR es igual a cero.

El asiento contable por el deterioro de los inventarios dependerá del tipo o motivo de la desvalorización de las existencias. Cuzcano (2020) considera cuatro situaciones posibles: 1) Pérdida de valor cuando el valor en libros supera al valor neto de realización, y se afectará el costo de ventas. 2) Pérdida por mermas en el proceso de producción, y el costo lo asume la producción. Práctica contable utilizada en Perú y aceptada por las autoridades tributarias. 3) Pérdidas por desmedros, productos malogrados, vencido su fecha de consumo o vencido comercialmente, por ser productos de la post-moda, y se afectará al costo de ventas. 4) Pérdida por robo, incendio u otros casos fortuitos, y se afectará a gastos operativos.

Para el primer método (Tabla 2, partida por partida), el asiento contable sería el siguiente:

Costo de ventas 3100 / A Inventarios 3100 (Ver tabla 2, método de partida por partida: Columna 6).

Se debe indicar que las prácticas contables en Perú, implican utilizar una cuenta de valuación distinta para el abono, denominada: Desvalorización de Inventarios, en lugar de la cuenta de inventarios involucrada.

\subsection{Revelación del deterioro de inventarios}

Los estados financieros y cualquier rubro contable requieren de explicaciones, sobre situaciones o hechos que generalmente no se contabilizan, como las garantías otorgadas mediante hipotecas, prendas, entre otros, o cuantificables (deterioro de valor de los inventarios) que implican la obligación moral de presentarlos, para que los usuarios o inversionistas, puedan tomar decisiones acertadas.

En el año 2015 las empresas mineras chilenas atravesaron una importante disminución de los precios de los minerales, situación que fue analizada por Orellana (2016) en un estudio exploratorio sobre 33 empresas en las memorias anuales del año 2015, el cual encontró que solo el $36 \%$ de ellas aplicaron algún test de deterioro a sus activos, propiedades, planta y equipo (PPE), intangibles o inventarios, con efectos en resultados, frente a un indicio de deterioro objetivo obtenido del mercado, es decir, los precios de los metales".

En el párrafo 2.36.b, de la NIC 2 Inventarios (IFRS Foundation, 2020b), se indica que debe revelarse en los Estados Financieros, los importes parciales y con ello, debe interpretarse como la anotación de los nombres de las cuentas y los valores con deterioro (párrafo 2.36.c). Igual en (párrafo 2.36.e) que regresa al (párrafo 2.34) que requiere las cuentas y conceptos del deterioro de valor de las existencias.

De acuerdo a lo señalado, las empresas deben detallar los conceptos e importes y del deterioro de valor de los inventarios. Como resultado de la revisión de la información financiera, las memorias y otros informes de las empresas industriales peruanas se pudo observar que son pocas las empresas industriales que revelan adecuadamente el deterioro de los inventarios. No se precisa la cuenta afectada, lo cual es una omisión a las revelaciones obligatorias en los reportes financieros. Incluso las instituciones que informan, son reservadas en las afirmaciones en los estados financieros, como los casos de ocho empresas, que detallan que sufrieron deterioros con los repuestos, envases y otros.

Revisando la información financiera de las empresas industriales del Perú, encontramos el 26\% (8 empresas) declaran los conceptos que involucran el deterioro de inventarios, pero no detallan los importes de cada cuenta, afectada por el deterioro. Además que el 74\% (23 empresas) no declaran los conceptos que involucran el deterioro de inventarios, tampoco detallan los importes de cada cuenta. Revelan montos globales. 
Sobre el valor neto realizable en la NIC 2 (IFRS Foundation, 2020c): “Generalmente, la rebaja hasta alcanzar el valor neto realizable se calcula para cada partida de los inventarios... No es apropiado realizar las rebajas a partir de partidas que reflejen clasificaciones completas de los inventarios, por ejemplo, sobre la totalidad de los productos terminados, o sobre todos los inventarios en un segmento de operación determinado". (Párrafo 2.29).

Como se puede reflexionar y comprender, la mayoría de las empresas industriales inscritas en la Bolsa de Valores de Lima, no están revelando adecuadamente los conceptos e importes relacionados con el deterioro de los inventarios.

\section{Metodología}

Se utilizó la información financiera (Estado de Situación Financiera), la económica (Estado de Resultados) y las notas a los estados financieros de las empresas industriales que se encuentran en la página WEB de la Bolsa de Valores de Lima para conocer el deterioro de valor de los inventarios y su efecto en los resultados de las empresas industriales, en el lapso 2017-2019. También se revisaron las Memorias Anuales de las empresas para verificar los conceptos por deterioro de inventarios.

\subsection{Población y muestra}

Para el presente trabajo de investigación se tomó como universo inicial a todas las empresas industriales que cotizaban en la Bolsa de Valores de Lima, 34 empresas industriales en promedio anual, excluyendo a las empresas de reciente ingreso o salida.

La población muestral del presente trabajo resultó de la aplicación de la siguiente formula estadística:
$\mathrm{n} \equiv$
$\mathrm{PQ} \quad .=$
$0.5(0.5)$
$=31$ empresas.
$(E / Z)^{2}+(P Q) / N$
$(0.05 / 1.96) 2+(0.25 / 34)$

\subsection{Técnicas e instrumentos de recolección y análisis de datos}

Se utilizó recolección de informacion documental. Se revisaron los reportes de la Bolsa de Valores de Lima, en lo referente a los estados financieros, dicha información, se incluyó en fichas u hojas para cada empresa.

Se estableció la correlación de Pearson, del deterioro de inventarios y la utilidad neta.

\section{Resultados y discusión}

\subsection{Deterioro de los inventarios y la incidencia en las ventas, utilidad neta y activos totales}

Los resultados obtenidos sobre el deterioro de los inventarios y su incidencia en la disminución de las utilidades netas de las empresas industriales del Perú, en el lapso 2017 - 2019, se muestran en las tablas 4, 5, 6 , 7 y 8. 
Tabla 4

Deterioro de Inventarios 2017

(en miles de dólares)

\begin{tabular}{|c|c|c|c|c|c|}
\hline Código & Empresas (industrias) & $\begin{array}{l}\text { Deterioro de } \\
\text { inventarios } \\
(\$ .)(000)\end{array}$ & \begin{tabular}{|l|} 
Deterioro Inventario (\% \\
de las ventas)
\end{tabular} & $\begin{array}{l}\text { Deterioro de } \\
\text { inventarios (\% de la } \\
\text { utilidad) }\end{array}$ & $\begin{array}{l}\text { Deterioro de } \\
\text { inventarios (\% del } \\
\text { activo) }\end{array}$ \\
\hline 1 & AGROINDUSTRIAS AIB & 0 & $0.00 \%$ & $0.00 \%$ & $0.00 \%$ \\
\hline 2 & ALLCORP SAA & 783 & $0.20 \%$ & $0.57 \%$ & $0.04 \%$ \\
\hline 3 & AUSTRAL GROUP & 465 & $1.05 \%$ & $7.17 \%$ & $0.15 \%$ \\
\hline 4 & CEMENTOS PACASMAYO S.AA & 916 & $1.19 \%$ & $3.16 \%$ & $0.11 \%$ \\
\hline 5 & CERVECERÍA SAN JUAN S.A. & 0 & $0.00 \%$ & $0.00 \%$ & $0.00 \%$ \\
\hline 7 & CONSORCIO INDUSTRIAL AREQUIPA & 0 & $0.00 \%$ & $0.00 \%$ & $0.09 \%$ \\
\hline 8 & CORPORACIÓN ACEROS AREQUIPA & 884 & $0.40 \%$ & $3.30 \%$ & $0.09 \%$ \\
\hline 9 & CORPORACIÓN CERAMICA & 1,883 & $12.94 \%$ & $38.76 \%$ & $1.91 \%$ \\
\hline 10 & CORPORACIÓN LINDLEY & 8,139 & $3.44 \%$ & $16.01 \%$ & $0.81 \%$ \\
\hline 11 & CREDITEX & 174 & $0.70 \%$ & $5.78 \%$ & $0.12 \%$ \\
\hline 12 & EDITORA EL COMERCIO S.A. & 913 & $1.65 \%$ & $11.16 \%$ & $0.23 \%$ \\
\hline 13 & ETNAS.A. & 114 & $0.69 \%$ & $7.11 \%$ & $0.12 \%$ \\
\hline 14 & EXSA S.A. & 250 & $0.48 \%$ & $7.28 \%$ & $0.12 \%$ \\
\hline 15 & ETERNITS.A & 108 & $0.40 \%$ & $2.08 \%$ & $0.09 \%$ \\
\hline 16 & GLORIAS.A & 3,916 & $1.24 \%$ & $6.16 \%$ & $0.40 \%$ \\
\hline 17 & HIDROSTALS.A & 464 & $3.50 \%$ & $33.21 \%$ & $0.52 \%$ \\
\hline 18 & INDECO S.A. & 382 & $0.65 \%$ & $4.09 \%$ & $0.28 \%$ \\
\hline 21 & COMPAÑÍA UNIVERSAL TEXTILS.A. & 1,301 & $20.38 \%$ & $15.61 \%$ & $2.73 \%$ \\
\hline 23 & EMPRESA SIDERURGICA DEL PERU S.A.A. & 2,337 & $1.88 \%$ & $5.40 \%$ & $0.47 \%$ \\
\hline 26 & INDUSTRIAS DEL ENVASES.A. & 0 & $0.00 \%$ & $0.00 \%$ & $0.00 \%$ \\
\hline 27 & INDUSTRIAS ELECTRO QUIMICAS S.A. (IEQSA) & 6 & $0.01 \%$ & $0.24 \%$ & $0.01 \%$ \\
\hline 28 & LAIVES.A. & 474 & $0.86 \%$ & $7.75 \%$ & $0.40 \%$ \\
\hline 30 & MANUFACTURA DE METALES Y ALUMINIO "RECORD" S.A. & 0 & $0.00 \%$ & $0.00 \%$ & $0.00 \%$ \\
\hline 32 & MICHELL Y CIA.S.A. & 0 & $0.00 \%$ & $0.00 \%$ & $0.00 \%$ \\
\hline 33 & MOTORES DIESEL ANDINOS S.A. & 502 & $1.59 \%$ & $5.28 \%$ & $0.28 \%$ \\
\hline 35 & PETROLEOS DEL PERÚ S.A. & 8 & $0.00 \%$ & $0.00 \%$ & $0.00 \%$ \\
\hline 37 & QUIMPAC S.A. & 3,110 & $4.63 \%$ & $10.52 \%$ & $0.47 \%$ \\
\hline 38 & REFINERIA LA PAMPILLA S.A.A. & 1,333 & $0.12 \%$ & $1.65 \%$ & $0.07 \%$ \\
\hline 40 & UNION DE CERVECERIAS PERUANAS BACKUS Y JOHNSTON S.A.A. & 1,116 & $0.28 \%$ & $0.26 \%$ & $0.07 \%$ \\
\hline 41 & UNIÓN ANDINA DE CEMENTOS S.A.A. -UNACEM S.A.A. & 2,676 & $1.53 \%$ & $1.87 \%$ & $0.10 \%$ \\
\hline 42 & YURAS.A. & 0 & $0.00 \%$ & $0.00 \%$ & $0.00 \%$ \\
\hline \multirow[t]{4}{*}{3} & TOTALES & 32,254 & $59.81 \%$ & $194.42 \%$ & $9.69 \%$ \\
\hline & 31 & & & & \\
\hline & PROMEDIO & 1,040 & $1.93 \%$ & $6.27 \%$ & $0.31 \%$ \\
\hline & TOTALES DE LOS RUBROS DECOMPARACIÓN & & $16,607,321$ & $1,380,162$ & $23,038,966$ \\
\hline
\end{tabular}

Fuente: Estados Financieros de la Bolsa de Valores de Lima.

Elaboración propia

El deterioro de los inventarios, en promedio representa en el año 2017 , el $6.27 \%$ de la utilidad neta y $1.93 \%$ de las ventas y $0.31 \%$ del total del activo. 
Tabla 5

Deterioro de Inventarios 2018

(en miles de dólares)

\begin{tabular}{|c|c|c|c|c|c|}
\hline Código & Empresas (industrias) & $\begin{array}{l}\text { Deterioro de } \\
\text { inventarios }(\$ .)(000)\end{array}$ & \begin{tabular}{|l|} 
Deterioro \\
Inventario (\% de las \\
ventas)
\end{tabular} & $\begin{array}{l}\text { Deterioro de } \\
\text { inventarios (\% de } \\
\text { la utilidad) }\end{array}$ & $\begin{array}{l}\text { Deterioro de } \\
\text { inventarios ( } \% \text { del } \\
\text { activo) }\end{array}$ \\
\hline 1 & AGROINDUSTRIAS AIB & 0 & $0.00 \%$ & $0.00 \%$ & $0.00 \%$ \\
\hline 2 & ALICORP SAA & 319 & $0.08 \%$ & $0.78 \%$ & $0.01 \%$ \\
\hline 3 & AUSTRAL GROUP & 493 & $0.86 \%$ & $5.10 \%$ & $0.14 \%$ \\
\hline 4 & CEMENTOS PACASMAYO S.AA & 963 & $1.27 \%$ & $13.56 \%$ & $0.11 \%$ \\
\hline 5 & CERVECERÍA SAN JUAN S.A. & 2 & $0.00 \%$ & $0.01 \%$ & $0.00 \%$ \\
\hline 7 & CONSORCIO INDUSTRIAL AREQUIPA & 23 & $0.44 \%$ & $3.29 \%$ & $0.14 \%$ \\
\hline 8 & CORPORACIÓN ACEROS AREQUIPA & 1,489 & $0.61 \%$ & $9.86 \%$ & $0.14 \%$ \\
\hline 9 & CORPORACIÓN CERAMICA & 89 & $0.60 \%$ & $73.10 \%$ & $0.10 \%$ \\
\hline 10 & CORPORACIÓN LINDLEY & 8,986 & $4.21 \%$ & $64.85 \%$ & $0.87 \%$ \\
\hline 11 & CREDITEX & 226 & $0.95 \%$ & $24.49 \%$ & $0.15 \%$ \\
\hline 12 & EDITORA EL COMERCIO S.A. & 829 & $1.68 \%$ & $83.53 \%$ & $0.18 \%$ \\
\hline 13 & ETNAS.A. & 35 & $0.19 \%$ & $2.42 \%$ & $0.04 \%$ \\
\hline 14 & EXSA S.A. & 2,435 & $4.26 \%$ & $86.90 \%$ & $0.94 \%$ \\
\hline 15 & ETERNIT S.A & 552 & $2.22 \%$ & $39.47 \%$ & $0.47 \%$ \\
\hline 16 & GLORIAS.A & 6,215 & $1.91 \%$ & $41.79 \%$ & $0.62 \%$ \\
\hline 17 & HIDROSTALS.A & 618 & $5.08 \%$ & $1197.60 \%$ & $0.67 \%$ \\
\hline 18 & INDECO S.A. & 205 & $0.36 \%$ & $8.24 \%$ & $0.15 \%$ \\
\hline 21 & COMPAÑÍA UNIVERSAL TEXTIL S.A. & 2,068 & $39.48 \%$ & $86.30 \%$ & $5.75 \%$ \\
\hline 23 & EMPRESA SIDERURGICA DEL PERU S.A.A. & 300 & $0.21 \%$ & $2.45 \%$ & $0.05 \%$ \\
\hline 26 & INDUSTRIAS DEL ENVASE S.A. & 29 & $0.24 \%$ & $5.29 \%$ & $0.05 \%$ \\
\hline 27 & INDUSTRIAS ELECTRO QUIMICAS S.A. (IEQSA) & 1 & $0.00 \%$ & $0.71 \%$ & $0.00 \%$ \\
\hline 28 & LAIVE S.A. & 347 & $0.61 \%$ & $17.51 \%$ & $0.27 \%$ \\
\hline 30 & MANUFACTURA DE METALES Y ALUMINIO "RECORD" S.A. & 0 & $0.00 \%$ & $0.00 \%$ & $0.00 \%$ \\
\hline 32 & MICHELL Y CIA. S.A. & 0 & $0.00 \%$ & $0.00 \%$ & $0.00 \%$ \\
\hline 33 & MOTORES DIESEL ANDINOS S.A. & 124 & $0.39 \%$ & $4.44 \%$ & $0.07 \%$ \\
\hline 35 & PETROLEOS DEL PERÚS.A. & 591 & $0.04 \%$ & $1.63 \%$ & $0.01 \%$ \\
\hline 37 & QUIMPACS.A. & 191 & $0.27 \%$ & $1.71 \%$ & $0.03 \%$ \\
\hline 38 & REFINERIA LA PAMPILLAS.A.A. & 10,797 & $0.89 \%$ & $61.63 \%$ & $0.53 \%$ \\
\hline 40 & UNION DE CERVECERIAS PERUANAS BACKUS Y JOHNSTON S.A.A. & 0 & $0.00 \%$ & $0.00 \%$ & $0.00 \%$ \\
\hline 41 & UNIÓN ANDINA DE CEMENTOS S.A.A. -UNACEM S.A.A. & 2,143 & $1.18 \%$ & $9.56 \%$ & $0.08 \%$ \\
\hline 42 & YURAS.A. & 184 & $0.20 \%$ & $0.85 \%$ & $0.02 \%$ \\
\hline & TOTALES & 40,254 & $68.23 \%$ & $1847.07 \%$ & $11.60 \%$ \\
\hline & 31 & & & & \\
\hline & PROMEDIO & 1,299 & $2.20 \%$ & $59.58 \%$ & $0.37 \%$ \\
\hline & TOTA LES DE LOS RUBROS DE COMPARACIÓN & & $18,308,866$ & $1,326,687$ & $25,837,086$ \\
\hline
\end{tabular}

Fuente: Estados Financieros de la Bolsa de Valores de Lima

Elaboración propia

El deterioro de los inventarios, en promedio representa en el año 2018 , el $59.58 \%$ de la utilidad neta y $2.20 \%$ de las ventas y $0.37 \%$ del total del activo. 
Tabla 6

Deterioro de Inventarios 2019

(en miles de dólares)

\begin{tabular}{|c|c|c|c|c|c|}
\hline Código & Empresas (industrias) & $\begin{array}{l}\text { Deterioro de } \\
\text { inventarios }(\$ .)(000)\end{array}$ & $\begin{array}{l}\text { Deterioro Inventario } \\
\text { (\% de las ventas) }\end{array}$ & \begin{tabular}{|l|} 
Deterioro de \\
inventarios (\% de la \\
utilidad)
\end{tabular} & $\begin{array}{l}\text { Deterioro de } \\
\text { inventarios (\% del } \\
\text { activo) }\end{array}$ \\
\hline 1 & AGROINDUSTRIAS AIB & 180 & $0.36 \%$ & $10.01 \%$ & $0.17 \%$ \\
\hline 2 & ALICORP SAA & 1,461 & $0.10 \%$ & $1.02 \%$ & $0.06 \%$ \\
\hline 3 & AUSTRAL GROUP & 229 & $0.12 \%$ & $3.51 \%$ & $0.07 \%$ \\
\hline 4 & CEMENTOS PACASMAYO S.AA & 491 & $0.18 \%$ & $1.24 \%$ & $0.06 \%$ \\
\hline 5 & CERVECERÍA SAN JUAN S.A. & 0 & $0.00 \%$ & $0.00 \%$ & $0.00 \%$ \\
\hline 7 & CONSORCIO INDUSTRIAL AREQUIPA & 27 & $0.16 \%$ & $1.22 \%$ & $0.15 \%$ \\
\hline 8 & CORPORACIÓN ACEROS AREQUIPA & 1,775 & $0.22 \%$ & $2.98 \%$ & $0.15 \%$ \\
\hline 9 & CORPORACIÓN CERAMICA & 38 & $0.08 \%$ & $0.92 \%$ & $0.04 \%$ \\
\hline 10 & CORPORACIÓN LINDLEY & 8,908 & $1.30 \%$ & $19.34 \%$ & $0.87 \%$ \\
\hline 11 & CREDITEX & 343 & $0.43 \%$ & $15.49 \%$ & $0.22 \%$ \\
\hline 12 & EDITORA EL COMERCIO S.A. & 233 & $0.16 \%$ & $0.76 \%$ & $0.05 \%$ \\
\hline 13 & ETNAS.A. & 0 & $0.00 \%$ & $0.00 \%$ & $0.00 \%$ \\
\hline 14 & EXSA S.A. & 958 & $0.49 \%$ & $42.05 \%$ & $0.37 \%$ \\
\hline 15 & ETERNITS.A & 292 & $0.34 \%$ & $5.99 \%$ & $0.25 \%$ \\
\hline 16 & GLORIAS.A & 4,460 & $0.43 \%$ & $10.16 \%$ & $0.46 \%$ \\
\hline 17 & HIDROSTAL S.A & 1,054 & $2.33 \%$ & $45.53 \%$ & $1.02 \%$ \\
\hline 18 & INDECO S.A. & 224 & $0.11 \%$ & $1.93 \%$ & $0.16 \%$ \\
\hline 21 & COMPAÑIA UNIVERSAL TEXTIL S.A. & 112 & $2.01 \%$ & $3.28 \%$ & $0.15 \%$ \\
\hline 23 & EMPRESA SIDERURGICA DEL PERU S.A.A. & 0 & $0.00 \%$ & $0.00 \%$ & $0.00 \%$ \\
\hline 26 & INDUSTRIAS DEL ENVASE S.A. & 67 & $0.16 \%$ & $2.49 \%$ & $0.12 \%$ \\
\hline 27 & INDUSTRIAS ELECTRO QUIMICAS S.A. (IEQSA) & 162 & $0.14 \%$ & $64.80 \%$ & $0.29 \%$ \\
\hline 28 & LAIVES.A. & 1,464 & $0.82 \%$ & $46.71 \%$ & $1.00 \%$ \\
\hline 30 & MANUFACTURA DE METALES Y ALUMINIO "RECORD" S.A. & 0 & $0.00 \%$ & $0.00 \%$ & $0.00 \%$ \\
\hline 32 & MICHELL Y CIA. S.A. & 0 & $0.00 \%$ & $0.00 \%$ & $0.00 \%$ \\
\hline 33 & MOTORES DIESEL ANDINOS S.A. & 138 & $0.12 \%$ & $3.05 \%$ & $0.08 \%$ \\
\hline 35 & PETROLEOS DEL PERÚ S.A. & 539 & $0.01 \%$ & $0.32 \%$ & $0.01 \%$ \\
\hline 37 & QUIMPAC S.A. & 682 & $0.35 \%$ & $11.02 \%$ & $0.10 \%$ \\
\hline 38 & REFINERIA LA PAMPILLAS.A.A. & 707 & $0.02 \%$ & $0.42 \%$ & $0.04 \%$ \\
\hline 40 & UNION DE CERVECERIAS PERUANAS BACKUS Y JOHNSTON S.A.A. & 0 & $0.00 \%$ & $0.00 \%$ & $0.00 \%$ \\
\hline 41 & UNIÓN ANDINA DE CEMENTOS S.A.A.A. UNACEM S.A.A. & 528 & $0.09 \%$ & $0.50 \%$ & $0.02 \%$ \\
\hline 42 & YURAS.A. & 772 & $0.26 \%$ & $1.22 \%$ & $0.07 \%$ \\
\hline \multirow[t]{4}{*}{31} & TOTALES & 25,844 & $10.79 \%$ & $295.96 \%$ & $6.01 \%$ \\
\hline & 31 & & & & \\
\hline & PROMEDIO & 834 & $0.35 \%$ & $9.55 \%$ & $0.19 \%$ \\
\hline & TOTA LES DE LOS RUBROS DE COMPARACIÓN & & $17,117,815$ & $1,287,501$ & $25,328,087$ \\
\hline
\end{tabular}

Fuente: Estados Financieros de la Bolsa de Valores de Lima Elaboración propia

El deterioro de los inventarios, en promedio representa en el año 2019 , el $9.55 \%$ de la utilidad neta y $0.35 \%$ de las ventas y $0.19 \%$ del total del activo.

En los tres años de estudio del deterioro de los inventarios, se aprecia que el rubro más afectado son las utilidades netas, mientras que las ventas y el total del activo reflejan una muy baja incidencia por dichos conceptos. 
Tabla 7

Resumen de resultados 2017 -2019

\begin{tabular}{|c|c|c|c|c|c|}
\hline EMPRESAS (INDUSTRI) & & $\begin{array}{l}\text { Deterioro de } \\
\text { inventarios }(\$ .)(000)\end{array}$ & $\begin{array}{l}\text { Deterioro Inventario } \\
\text { (\% de las ventas) }\end{array}$ & $\begin{array}{l}\text { Deterioro de } \\
\text { inventarios (\% de la } \\
\text { utilidad) }\end{array}$ & $\begin{array}{l}\text { Deterioro de } \\
\text { inventarios (\% del } \\
\text { activo) }\end{array}$ \\
\hline 2017 & & 32,254 & $59.81 \%$ & $194.42 \%$ & $9.69 \%$ \\
\hline 2018 & & 40,254 & $68.23 \%$ & $1847.07 \%$ & $11.60 \%$ \\
\hline 2019 & & 25,844 & $10.79 \%$ & $295.96 \%$ & $6.01 \%$ \\
\hline TOTAL & & 98,352 & $138.83 \%$ & $2337.45 \%$ & $27.30 \%$ \\
\hline "Promedio (31) & 31 & 3173 & $4.5 \%$ & $75.4 \%$ & $0.9 \%$ \\
\hline
\end{tabular}

Fuente: Estados Financieros de la Bolsa de Valores de Lima. Elaboración propia.

El impacto más importante del deterioro de los inventarios se presentó en las utilidades netas del período, es de $75.4 \%$, en promedio, lo cual redujo las utilidades a repartir a los propietarios de las empresas.

\subsection{Correlación de las variables}

En la tabla 8 se presentan los resultados de la correlación entre el inventario deteriorado y su relación con la utilidad neta, en donde se puede apreciar que existe una alta correlación ( 21 empresas) y solamente ocho empresas tienen muy baja correlación, aparte, dos empresas no tuvieron deterioro de los inventarios.

Nuestra hipótesis general ha sido validada parcialmente. La tabla 8, evidencia que 21 empresas (67.7\%) denotan una elevada correlación de las variables, y solamente 8 empresas (25.8\%), tienen una baja correlación, debido a la alta variabilidad de los importes monetarios por el deterioro. Sin embargo, cuando trabajamos con los promedios obtenidos, apreciamos que el gasto por el deterioro del inventario de todas las empresas ascienda al $4.5 \%$ de las ventas netas o $75.4 \%$ de las utilidades netas, o al $0.9 \%$ de los activos totales de las empresas industriales. Se puede afirmar, que el deterioro de los inventarios medido como parte de las ventas o de los activos totales, es operacionalmente manejable. Sin embargo, cuando los medimos como parte de las utilidades netas, se aprecia un importante efecto en la disminución de las utilidades de todas las empresas industriales peruanas. En ese sentido, cuando se analicen los estados financieros, se debería incluir la evaluación del deterioro de los inventarios y cómo y cuánto afectó. 
Tabla 8

Correlación del deterioro de inventarios

y la utilidad neta 2017- 2019

\begin{tabular}{|c|c|c|c|c|c|c|c|c|c|c|}
\hline Código & EMPRESAS (INDUSTRIAS) & & & $\begin{array}{l}\text { Deterioro } \\
\text { inventario - } 2017 \\
\text { \$. }(000)\end{array}$ & $\begin{array}{l}\text { Deterioro } \\
\text { inventario-2018 } \\
(\$ .)(000)\end{array}$ & $\begin{array}{l}\text { Deterioro } \\
\text { inventario - } 2019 \\
(\$ .)(000) \\
\end{array}$ & $\begin{array}{l}\text { Deterioro } \\
\text { inventarios }(\% \text { de } \\
\text { la utilidad- 2017) }\end{array}$ & $\begin{array}{l}\text { Deterioro } \\
\text { inventarios (\% de la } \\
\text { utilidad-2018) }\end{array}$ & $\begin{array}{l}\text { Deterioro } \\
\text { inventarios (\% de } \\
\text { la utilidad-2019) }\end{array}$ & $\begin{array}{l}\text { Coeficiente } \\
\text { correlación }\end{array}$ \\
\hline 1 & AGROINDUSTRIAS AIB & & & 0 & 0 & 180 & $0.00 \%$ & $0.00 \%$ & $10.01 \%$ & -1.00 \\
\hline 2 & ALICORP SAA & & & 783 & 319 & 1,461 & $0.57 \%$ & $0.78 \%$ & $1.02 \%$ & 0.62 \\
\hline 3 & AUSTRAL GROUP & & & 465 & 493 & 229 & $7.17 \%$ & $5.10 \%$ & $3.51 \%$ & 0.77 \\
\hline 4 & \multicolumn{2}{|l|}{ CEMENTOS PACASMAYOS.AA } & & 916 & 963 & 491 & $3.16 \%$ & $13.56 \%$ & $1.24 \%$ & 0.69 \\
\hline 5 & \multicolumn{2}{|l|}{ CERVECERÍA SAN JUANS.A. } & & 0 & 2 & 0 & $0.00 \%$ & $0.01 \%$ & $0.00 \%$ & 0.00 \\
\hline 7 & \multicolumn{2}{|c|}{ CONSORCIO INDUSTRIAL AREQUIPA } & & 0 & 23 & 27 & $0.00 \%$ & $3.29 \%$ & $1.22 \%$ & 0.69 \\
\hline 8 & \multicolumn{2}{|c|}{ CORPORACIÓN ACEROS AREQUIPA } & & 884 & 1,489 & 1,775 & $3.30 \%$ & $9.86 \%$ & $2.98 \%$ & 0.16 \\
\hline 9 & \multicolumn{2}{|l|}{ CORPORACIÓN CERAMICA } & & 1,883 & 89 & 38 & $38.76 \%$ & $73.10 \%$ & $0.92 \%$ & 0.05 \\
\hline 10 & CORPORACIÓN LINDLEY & & & 8,139 & 8,986 & 8,908 & $16.01 \%$ & $64.85 \%$ & $19.34 \%$ & 0.62 \\
\hline 11 & CREDITEX & & & 174 & 226 & 343 & $5.78 \%$ & $24.49 \%$ & $15.49 \%$ & 0.32 \\
\hline 12 & \multicolumn{2}{|l|}{ EDITORA EL COMERCIOS.A. } & & 913 & 829 & 233 & $11.16 \%$ & $83.53 \%$ & $0.76 \%$ & 0.50 \\
\hline 13 & ETNAS.A. & & & 114 & 35 & 0 & $7.11 \%$ & $2.42 \%$ & $0.00 \%$ & 1.00 \\
\hline 14 & EXSA S.A. & & & 250 & 2,435 & 958 & $7.28 \%$ & $86.90 \%$ & $42.05 \%$ & 0.99 \\
\hline 15 & ETERNITS.A & & & 108 & 552 & 292 & $2.08 \%$ & $39.47 \%$ & $5.99 \%$ & 0.95 \\
\hline 16 & GLORIAS.A & & & 3,916 & 6,215 & 4,460 & $6.16 \%$ & $41.79 \%$ & $10.16 \%$ & 0.99 \\
\hline 17 & HIDROSTALS.A & & & 464 & 618 & 1,054 & $33.21 \%$ & $1197.60 \%$ & $45.53 \%$ & -0.26 \\
\hline 18 & INDECOS.A. & & & 382 & 205 & 224 & $4.09 \%$ & $8.24 \%$ & $1.93 \%$ & -0.27 \\
\hline 21 & \multicolumn{2}{|c|}{ COMPAÑÍA UNIVERSAL TEXTILS.A. } & & 1,301 & 2,068 & 112 & $15.61 \%$ & $86.30 \%$ & $3.28 \%$ & 0.87 \\
\hline 23 & \multicolumn{2}{|c|}{ EMPRESA SIDERURGICA DEL PERUS.A.A. } & & 2,337 & 300 & 0 & $5.40 \%$ & $2.45 \%$ & $0.00 \%$ & 0.94 \\
\hline 26 & \multicolumn{2}{|l|}{ INDUSTRIAS DEL ENVASES.A. } & & 0 & 29 & 67 & $0.00 \%$ & $5.29 \%$ & $2.49 \%$ & 0.40 \\
\hline 27 & \multicolumn{2}{|c|}{ INDUSTRIAS ELECTRO QUIMICASS.A. } & & 6 & 1 & 162 & $0.24 \%$ & $0.71 \%$ & $64.80 \%$ & 1.00 \\
\hline 28 & LAIVES.A. & & & 474 & 347 & 1,464 & $7.75 \%$ & $17.51 \%$ & $46.71 \%$ & 0.94 \\
\hline 30 & \multicolumn{2}{|c|}{ MANUFACTURA DE METALES Y ALUMINIO "RECORD"S.A. } & & 0 & 0 & 0 & $0.00 \%$ & $0.00 \%$ & $0.00 \%$ & 0.00 \\
\hline 32 & MICHELL Y CIA.S.A. & & & 0 & 0 & 0 & $0.00 \%$ & $0.00 \%$ & $0.00 \%$ & 0.00 \\
\hline 33 & \multicolumn{2}{|c|}{ MOTORES DIESEL ANDINOSS.A. } & & 502 & 124 & 138 & $5.28 \%$ & $4.44 \%$ & $3.05 \%$ & 0.77 \\
\hline 35 & PETROLEOS DEL PERÚS.A. & & & 8 & 591 & 539 & $0.00 \%$ & $1.63 \%$ & $0.32 \%$ & 0.71 \\
\hline 37 & QUIMPACS.A. & & & 3,110 & 191 & 682 & $10.52 \%$ & $1.71 \%$ & $11.02 \%$ & 0.59 \\
\hline 38 & \multicolumn{2}{|c|}{ REFINERIA LA PAMPILLAS.A.A. } & & 1,333 & 10,797 & 707 & $1.65 \%$ & $61.63 \%$ & $0.42 \%$ & 1.00 \\
\hline 40 & \multicolumn{2}{|c|}{ UNION DE CERVECERIAS PERUANAS BACKUS Y JOHNSTONS.A.A. } & & 1,116 & 0 & 0 & $0.26 \%$ & $0.00 \%$ & $0.00 \%$ & 1.00 \\
\hline 41 & \multicolumn{2}{|c|}{ UUNIÓN ANDINA DE CEMENTOSS.A.A. UNACEM S.A.A. } & & 2,676 & 2,143 & 528 & $1.87 \%$ & $9.56 \%$ & $0.50 \%$ & 0.41 \\
\hline 42 & YYRAS.A. & & & 0 & 184 & 772 & $0.00 \%$ & $0.85 \%$ & $1.22 \%$ & 0.87 \\
\hline & TOTALES & & & 32,254 & 40,254 & 25,844 & $194.42 \%$ & $1847.07 \%$ & $295.96 \%$ & 16 \\
\hline & 31 & & 31 & & & & & & & \\
\hline & PROMEDIO & PROMEDIO & & 1,040 & 1,299 & 834 & $6.27 \%$ & $59.58 \%$ & $9.55 \%$ & 0.52653331 \\
\hline
\end{tabular}

Fuente: Estados Financieros de la Bolsa de Valores de Lima

Elaboración propia

\subsection{Discusión}

La Norma Internacional de contabilidad N²-Inventarios, determina que el importe de cualquier reversión de la rebaja de valor que resulte de un incremento en el valor neto realizable, se reconocerá como una reducción en el valor de los inventarios que hayan sido reconocidos como gasto en el periodo en que la recuperación del valor tenga lugar.

En lo referente al impacto del deterioro de los inventarios en los resultados económicos y rentabilidad, coincidimos con Jiménez \& Rivera (2019), sin embargo, no hemos profundizado en el tema tributario.

Igualmente, consideramos que la desvalorización de los inventarios incide en la rentabilidad de las empresas, tal como lo afirman: Pacheco, Brancacho y Galarza (2017). En ese sentido, recordemos que la estimación del 
deterioro de los inventarios implica reconocer el gasto correspondiente, lo cual incide en la disminución de las utilidades y de la rentabilidad, lo que podemos apreciar en los resultados de la presente investigación.

Orellana (2016) afirma, que solo el $36 \%$ de las empresas mineras chilenas aplicaron algún test de deterioro a sus activos PPE. El fundamento teórico que desarrollamos en el presente estudio, es más preciso, respecto a lo que establecen las normas internacionales de contabilidad y los temas de revelaciones que se están omitiendo. En ese sentido, encontramos a las empresas industriales peruanas, que no detallaron las cuentas de inventarios que sufrieron deterioro (no se precisa: ¿productos terminados o materias primas, o envases, o repuestos o suministros?) ; ello es una omisión de las revelaciones, de parte de los responsables de la alta dirección de las empresas industriales del Perú.

Las normas internacionales de contabilidad, requieren que se revele información adicional en los estados financieros, cuando ayude a comprender al lector y evite la "omisión o expresión inadecuada de esa información" o que parezca que se "oculta información material o con importancia relativa".(párrafo 1.7)

Se complementa con lo siguiente: "Al decidir si una determinada política contable debe revelarse, la gerencia considerará si la revelación ayudaría a los usuarios a comprender la forma en la que las transacciones y otros sucesos y condiciones se reflejan en la información sobre el rendimiento y la situación financiera". (párrafo 1.119).

Indudablemente que la información detallada de los rubros o cuentas contables que sufrieron deterioro de valor, serán altamente relevantes, para el inversor o los lectores especializados.

Como se puede reflexionar y comprender, la mayoría de las empresas industriales inscritas en la Bolsa de Valores de Lima, no están revelando adecuadamente los conceptos e importes relacionados con el deterioro de los inventarios.

En el contexto descrito, la investigación efectuada nos permitió conocer cómo incide la norma internacional de contabilidad $\mathrm{N}^{\circ} 2$-Inventarios, en la aplicación y determinación del deterioro de existencias en las empresas industriales del Perú.

\section{Conclusiones}

Encontramos en ésta investigación, que existe una correlación muy fuerte de 21 empresas, entre el deterioro de inventarios y la disminución de la utilidad neta de las empresas industriales de Perú (tabla 8). Es un resultado lógico, pues el deterioro, es un gasto empresarial. La correlación promedio es de 0.53 , que resulta menor, por ocho empresas con baja correlación y dos empresas que declaran no tener problemas con el deterioro de inventarios. Sin embargo, las empresas con muy buena correlación, representan el $67.7 \%(21 / 31=67.7 \%)$ y tienen una correlación promedio de 0.83 .

El efecto del gasto por el deterioro del inventario de las empresas, en promedio, fue de $4.5 \%$ de las ventas netas o $75.4 \%$ de las utilidades netas de las empresas industriales, o el $0.9 \%$ de los activos totales.

El deterioro de los inventarios tiene un impacto importante en la disminución de las utilidades netas, y paralelamente, en las disminución de la utilidades a repartir a los accionistas de la empresas industriales del Perú.

Por último, las empresas industriales deben mejorar la comunicación o revelación del deterioro de inventarios, en los estados financieros, detallando las cuentas y los importes monetarios, para que los lectores de la información contable, puedan analizar adecuadamente y tomar decisiones financieras que correspondan al contexto. 


\section{Referencias bibliográficas}

Abanto Bromley, Marta. (2016). Revista Contadores y Empresas de la 2da, quincena de enero 2016. Perú.

Ataupillco, Victor (2020). NIIF. Taxonomía e Impuestos. PCGE. Incluye Efectos Del Covid 19. Tomos I y II. Ediciones Desarrollo Contable S.A.C. Lima, Perú.

Benavides Villacís, Mayra Valeria. (2017). “El Tratamiento Contable del Deterioro del Inventario en la Empresa Electro Irmavi. Pontificia Universidad Católica del Ecuador.

Bernia Pacheco de León, Flor Marleny; Brancacho Alejos, Laura Ysabel; Galarza Palacios, Janet Ruth. (2017). La Desvalorización de las Existencias, Pérdida de Materia Prima y su Incidencia en la Rentabilidad de la Empresa Constructora Eital Edificaciones Sac. Año 2015 - 2016. Universidad Peruana De Las Américas. http://repositorio.ulasamericas.edu.pe/handle/upa/325.

Campoverde Pacheco, M. S., \& Orellana Castillo, A. F. (2012). Análisis del deterioro del valor de los inventarios (Bachelor'sthesis). Retrievedfromhttp://dspace.ucuenca.edu.ec/handle/123456789/1285.

Cuzcano C.,Alex. (2020). El Amauta de las NIIF. Editora Global CPA School S.A.C. Lima.

Del Campo Moreno, Paloma. (2019). Contabilidad Financiera. Normas de Registro y Valoración. Editores: Sanz y Torres: UNED, Universidad Nacional de Educación a Distancia. Madrid, España.

Ferrer Quea, Alejandro. Mermas y Desmedros. Criterios Contables y Tributarios Actualidad Empresarial N²16, 2010, octubre. Págs. IV-1-IV-7.

Fonseca, J. J. \& Murillo, B. E. (2015). Análisis financiero en la valoración del deterioro generado por la aplicación de la NIC 2 mercado de la construcción sector retail. Recuperado de:

http://hdl.handle.net/20.500.12010/2531.

Garizabal, Luz E.; Barrios, Ibeth P.; Bernal, Omaira y Garizabal, Mildred E. (2020) Estrategias de planeación tributaria para optimizar impuesto de los contribuyentes. Revista Espacios.Vol. 41 (№ 08) Año 2020. Pág. 1-8. Recuperado de: revistaespacios.com/a20v41n08/a20v41n08p01.pdf.

Guajardo Cantú, Gerardo y Andrade de Guajardo, Nora. (2019.págs. 381 y382). Contabilidad Financiera. Editorial McGraw Hill. México.

Jiménez Barreto, A. D., \& Rivera Capcha, E. (2019). Mermas y Desmedros- Impacto financiero y tributario en las empresas del sector manufactura (actividades de impresión) en la Zona Este y Lima, 2017. Universidad Peruana de Ciencias Aplicadas (UPC), Lima, Perú. https://doi.org/10.19083/tesis/625958.

Orellana Fuentes, C. (2016). Deterioro del valor de los activos mineros según las normas internacionales de contabilidad y la gestión de resultados: evidencia para las empresas mineras chilenas. CAPIC REVIEW, 14, 83-92.Recuperado de: https://doi.org/10.35928/cr.vol14.2016.35

Soto-Restrepo, B. A., Quiros-Jaramillo, J. de la C., \& Mesa-Velásquez, G. S. (2014). Análisis comparativo del tratamiento contable y financiero de los inventarios entre NIC 2 plena, NIIF pymes sección 13 y Decreto 2706 capítulo 8. Cuadernos De Contabilidad, 14(36). pp. 903-941. Recuperado a partir de https://revistas.javeriana.edu.co/index.php/cuacont/article/view/7908.

The International Financial Reporting Standards Foundation (IFRS Foundation). El Marco Conceptual para la Información Financiera (el Marco Conceptual) (66 págs.) fue emitido por el Consejo de Normas Internacionales de Contabilidad en septiembre de 2018. Recuperado de: 
https://www.mef.gob.pe/contenidos/conta_publ/con_nor_co/vigentes/nic/RedBV2018_ConceptualFram ework_GVT.pdf. 02.11.2020.

The International Financial Reporting Standards Foundation (IFRS Foundation). NIC 1 Presentación de Estados Financieros (25 págs.). Actualizada por el Consejo de Normas Internacionales de Contabilidad en enero de 2020. Recuperado de:

https://www.mef.gob.pe/contenidos/conta_publ/con_nor_co/nic/SpanishRed2020_IAS01_GVT.pdf. 10.12 .20

The International Financial Reporting Standards Foundation (IFRS Foundation). NIC 2 Inventarios (06 págs.). Actualizada por el Consejo de Normas Internacionales de Contabilidad en enero de 2020. Recuperado de: https://www.mef.gob.pe/contenidos/conta_publ/con_nor_co/nic/SpanishRed2020_IAS02_GVT.pdf. 09.12.2020.

Esta obra está bajo una Licencia Creative Commons Attribución-NoCommercial 4.0 International

(cc) BY-NC 